11,12

\title{
Критическая температура трехвершинной модели Поттса на решетке Кагоме
}

\author{
(C) А.Б. Бабаев ${ }^{1,2}$, А.К. Муртазаев 1,2 \\ ${ }^{1}$ Институт фризики им. Х.И. Амирханова ДагНЦ РАН, \\ Махачкала, Россия \\ 2 Дагестанский научный центр РАН, \\ Махачкала, Россия \\ E-mail: b_albert78@mail.ru \\ Поступила в Редакцию 26 февраля 2019 г. \\ В окончательной редакции 26 февраля 2019 г. \\ Принята к публикации 26 февраля 2019 г.
}

Рассматривается модель Поттса на решетке Кагоме. Методом Монте-Карло получены температурные зависимости термодинамических параметров теплоемкости $C$, параметра порядка $m$ и восприимчивости $\chi$. Расчеты проводились для систем с периодическими граничными условиями. Рассматривались системы с линейными размерами $L \times L=N, L=20-90$. На основе метода кумулянтов Биндера четвертого порядка рассчитана критическая температура $\left(T_{c}\right)$ для трехвершинной модели Поттса на решетке Кагоме. Показано, что полученное значение $T_{c}$ в пределах статистической ошибки находится в хорошем согласии с результатами полученных методами трансфер-матриц и полиномиального приближения.

Ключевые слова: Модель Поттса, решетка Кагоме, критическая температура, Монте-Карло.

DOI: $10.21883 /$ FTT.2019.07.47848.400

\section{1. Введение}

Одной из часто используемых моделей в статистической физике является модель Поттса, которая была предложена в 1952 г. Домбом [1] и является теоретическим инструментом, применяемым для изучения широкого класса явлений и объектов в физике конденсированных сред.

Несмотря на интенсивные теоретические исследования двумерных спиновых решеточных систем, описываемых моделями Поттса, в течение последних пятидесяти лет, к настоящему времени существует совсем немного надежно установленных фактов. Известно, что в чистой модели Поттса с состоянием $q>q_{c}(D)$, где $D$-размерность системы, наблюдается ФП первого рода, а ФП второго рода в случае $q<q_{c}(d)$ [2,3]. Для 2D модели Поттса величина $q_{c}(d=2)=4$, в то время как для 3D модели $q c(d=3)=2.45[1,3]$. Причем для $q_{c}(d=2)=4$ наблюдается ФП второго рода, а для $q_{c}(d=3)=2.45$ - слабо выраженный ФП первого рода. Кроме того, для двумерных моделей Поттса с числом состояний спина $q$ на квадратной, треугольной и гексагональных решетках были получены простые полиномиальные выражения [4], позволяющие оценить точное значение критической температуры. Справедливость этих выражений строго была доказана для ферромагнитных моделей Поттса с $q \geq 4$ и $q=2$ [5]. Отдельный интерес представляет модель Поттса на решетке Кагоме [6] связанный с многообразием ее структурных свойств. В качестве примера вещества обладающего структурой решетки Кагоме можно приве- сти минерал - гербертсмитит с химической формулой $\mathrm{ZnCu}_{3}(\mathrm{OH})_{6} \mathrm{Cl}_{2}$ [7]. Решетка Кагоме является одной из простых двумерных структур, принадлежащих к Архимедовым решеткам. Для случая $q=2$ эта модель была решена точно более пятидесяти лет тому назад [8]. В то же время при попытках рассчитать критические параметры для трехвершинной модели Поттса на решетке Кагоме аналитические методы сталкиваются с непреодолимыми трудностями, что привело к разработке классических гипотез, позволяющих приблизительно оценить значение критической температуры, справедливость которых в последнее десятилетие не доказана для случая с $q=3$ [9]. В связи с этим целью настоящей работы является исследование методом Монте-Карло (МК) термодинамических свойств модели Поттса с числом состояний спина $q=3$ на решетке Кагоме, вычисление критической температуры и сравнение полученных данных для этой модели с данными аналитических методов.

\section{2. Двумерная трехвершинная модель Поттса на решетке Кагоме}

Ферромагнитная (ФМ) модель Поттса представляет собой естественное обобщение модели Изинга. В модели Изинга имеется $N$ дискретных объектов, называемых узлами решетки, каждый из которых может находиться в одном из двух состояний. В модели Поттса каждый узел может находиться уже в одном из $q \geq 2$ состояний. Поэтому, при построении двумерной ферромагнитной модели Поттса с числом состояний спина $q=3$ на 


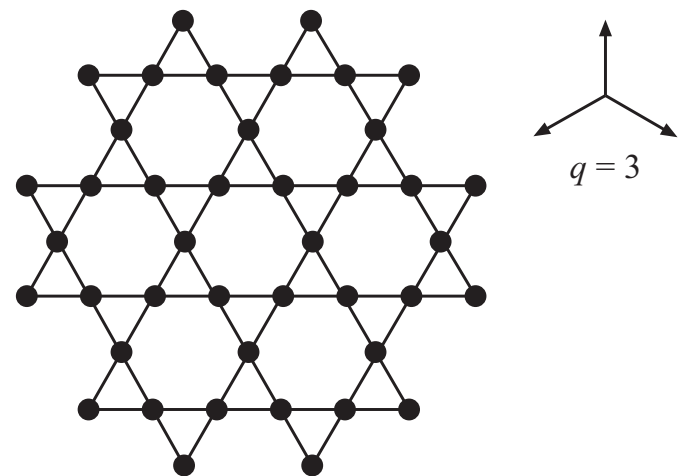

Рис. 1. Двумерная трехвершинная модель Поттса на решетке Кагоме.

решетке Кагоме необходимо иметь в виду следующие особенности [1]:

1) в узлах решетки Кагоме расположены спины $S_{i}$, которые могут находиться в одном из $q \geq 2$ состояний (см. рис. 1). Заметим, что решетка Кагоме представляет собой треугольную решетку, из которой регулярным образом удалена четверть узлов;

2) энергия парного взаимодействия принимает одно значение, если взаимодействующие узлы находятся в одинаковых состояниях (безразлично в каких именно), и другое значение, если они находятся в разных состояниях (опять же, все равно в каких именно).

С учетом этих особенностей микроскопический гамильтониан такой системы может быть представлен в виде [1]:

$$
H=-\frac{J}{2} \sum_{i, j} \cos \theta_{i, j}
$$

где $J$ - параметр обменного ферромагнитного взаимодействия $(J>0)$ между ближайшими соседями, $\theta_{i, j}-$ угол между взаимодействующими спинами $S_{i}-S_{j}$, причем $\theta_{i, j}=\frac{2 \pi n}{q}, n=0,1,2, \ldots, q-1$ и при $q=3$ принимает три значения $0^{\circ}, 120^{\circ}$ и $240^{\circ}$. Модель Поттса с гамильтонианом вида (1) также хорошо описывает физические свойства многих упорядоченных и неупорядоченных структур (см. [10-13]).

При компьютерном моделировании нами использовался кластерный алгоритм Вольфа метода МК. При этом на каждом МК шаге сначала строится кластер, затем его переворачивают. Более подробно этот алгоритм рассмотрен в работе [15]. Начальные конфигурации задавались таким образом, чтобы все спины находились в одинаковых состояниях. Для вывода системы в равновесное состояние вычислялось время релаксации $\tau_{0}$ для всех систем с линейными размерами $L$. Этот неравновесный участок отбрасывали. Затем усреднение проводилось по участку марковской цепи длиной $\tau=190 \tau_{0}$. Для самой большой системы $L=90, \tau_{0}=2 \cdot 10^{8} \mathrm{MK}$ step/spin. Кроме того, для повышения точности расчетов проводилось усреднение по 15-ти различным начальным конфигурациям. Затем эти данные использовались для расчета средних значений термодинамических параметров.

\section{3. Результаты численного эксперимента}

Для наблюдения за температурным ходом поведения теплоемкости и восприимчивости использовались флуктуационные соотношения [16]:

$$
\begin{aligned}
& C=\left(N K^{2}\right)\left(\left\langle U^{2}\right\rangle-\langle U\rangle^{2}\right), \\
& \chi=(N K)\left(\left\langle m_{F}^{2}\right\rangle-\left\langle m_{F}\right\rangle^{2}\right),
\end{aligned}
$$

где $K=|J| / k_{\mathrm{B}} T, N=0.75 L^{2}-$ число магнитных узлов, $U-$ внутренняя энергия, $m_{F}-$ параметр порядка системы, угловые скобки обозначают усреднение по ансамблю. В качестве намагниченности $\left(m_{F}\right)$, для ФМ модели Поттса использовалось следующее выражение $[17,18]$

$$
m_{F}=\left\langle\frac{3}{2} \sum_{\alpha=1}^{3}\left(\frac{N_{\alpha}}{N}-\frac{1}{3}\right)^{2}\right\rangle^{1 / 2},
$$

где $N_{\alpha}=\left\{N_{1}, N_{2}, N_{3}\right\}, N_{1}$ - число спинов в состоянии с $q=1, N_{2}$ - число спинов в состоянии с $q=2, N_{3}-$ число спинов в состоянии с $q=3$.

На рис. 2 и 3 представлены характерные зависимости для восприимчивости $\chi$ и теплоемкости $C$ от температуры $T$ для двумерной трехвершинной ФМ модели Поттса на решетке Кагоме для систем с линейными размерами $L=10-90$. Здесь и далее на всех рисунках погрешность данных не превышает размеров символов, используемых для построения графиков. Отметим, что в зависимости восприимчивости $\chi$ и теплоемкости $C$ от температуры для всех исследуемых нами систем проявляются четко выраженные максимумы, и эти максимумы в пределах погрешности приходят на одну температуру.

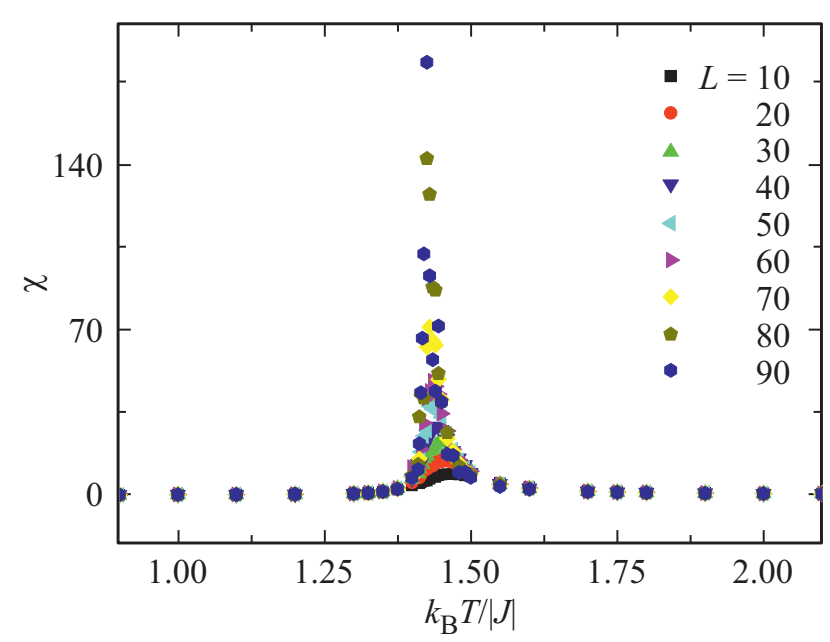

Рис. 2. Температурная зависимость восприимчивости $\chi$ для двумерной трехвершинной модели Поттса на решетке Кагоме. 
Критическая температура двумерной терхвершинной модели Поттса на решетке Кагоме

\begin{tabular}{c|c|c|c|c}
\hline \multirow{2}{*}{ Модель } & \multicolumn{4}{|c}{ Метод } \\
\cline { 2 - 5 } & $\begin{array}{c}\text { Монте-Карло } \\
\text { (наши данные) }\end{array}$ & $\begin{array}{c}\text { Низкотемпературное } \\
\text { разложение }[20]\end{array}$ & $\begin{array}{c}\text { Трансфер-матриц } \\
{[9]}\end{array}$ & $\begin{array}{c}\text { Классическия гипотеза } \\
\text { (Wu conjecture) }[4]\end{array}$ \\
\hline Вершинная & 1.4186 & 1.41909 & 1.41975 & 0.6912 \\
Обычная & - & 0.94606 & 0.9465 &
\end{tabular}

На рис. 4 представлены температурные зависимости намагниченности $m_{F}$ для двумерной трехвершинной модели Поттса на решетке Кагоме. Как видно из рис. 4 наблюдается монотонное уменьшение величины $m_{F}$ с ростом температуры и заметное уменышение высокотемпературных „хвостов“ при увеличении линейного размера $L$.

Для определения критических температур и анализа характера фазового перехода использовался метод куму-

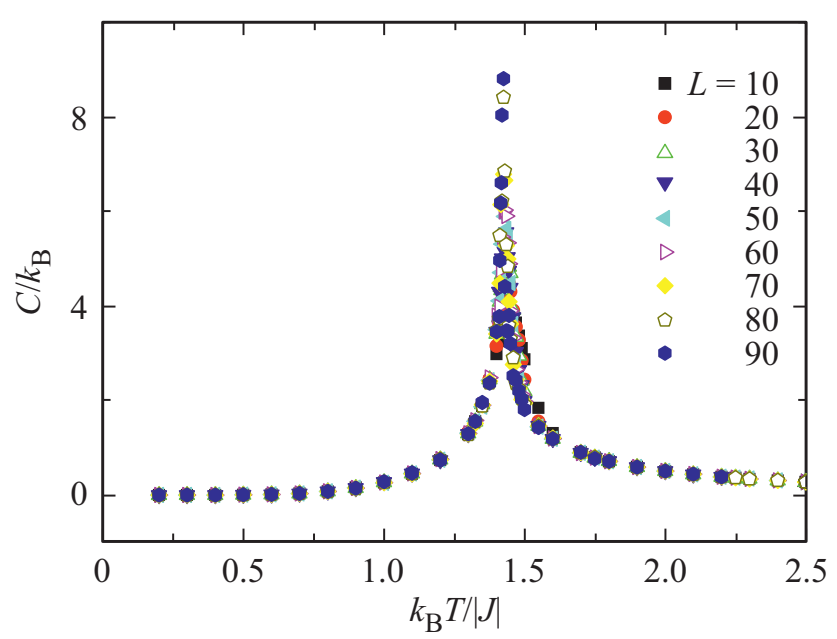

Рис. 3. Температурная зависимость теплоемкости $C$ для двумерной трехвершинной модели Поттса на решетке Кагоме.

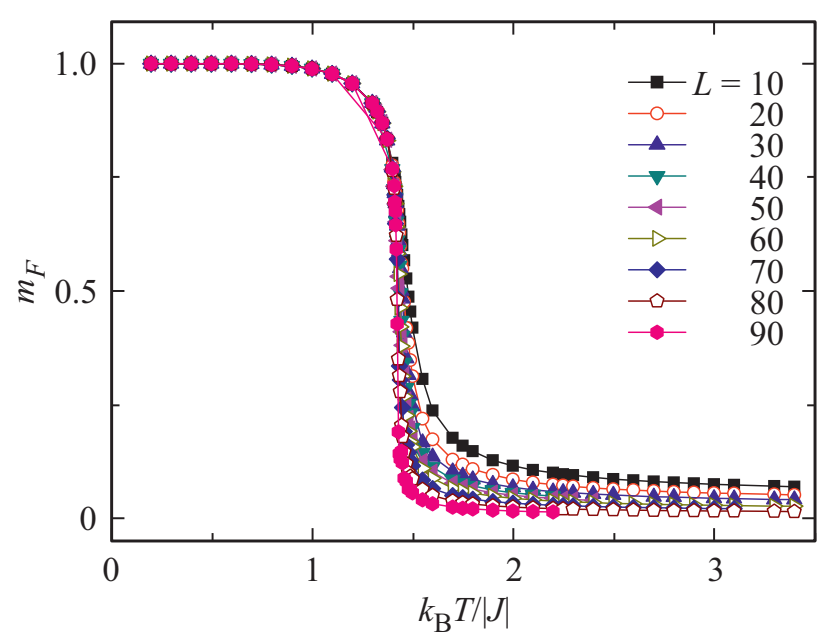

Рис. 4. Температурная зависимость намагничеснности $m_{F}$ для двумерной трехвершинной модели Поттса на решетке Кагоме.

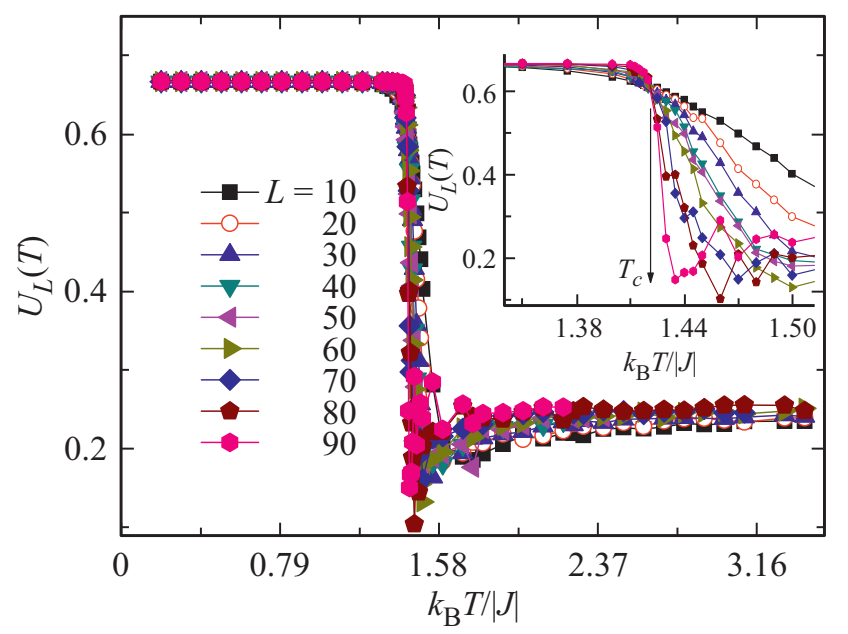

Рис. 5. Температурная зависимость кумулянтов Биндера $U_{L}(T)$ для двумерной трехвершинной модели Поттса на решетке Кагоме. На вставке показана точка пересечения кумулянтов Виндера $U_{L}(T)$, соответствующая критической температуре $T_{C}$.

лянтов Биндера четвертого порядка [19]:

$$
\begin{gathered}
V_{L}(T)=1-\frac{\left\langle E^{4}\right\rangle_{L}}{3\left\langle E^{2}\right\rangle_{L}^{2}}, \\
U_{L}(T)=1-\frac{\left\langle m^{4}(T, L)\right\rangle_{L}}{3\left\langle m^{2}(T, L)\right\rangle_{L}^{2}},
\end{gathered}
$$

где $E-$ энергия и $m-$ параметр порядка системы с линейными размерами $L$. Выражения (5) и (6) позволяют с хорошей точностью определить температуру фазового перехода $T_{l}$ при фазовых переходах первого и второго рода соответственно.

Следует отметить, что применение кумулянтов Биндера позволяет также хорошо тестировать тип фазового перехода в системе. В случае ФП второго рода кривые температурной зависимости кумулянтов Биндера $U_{L}(T)$ имеют четко выраженную точку пересечения. Характерные зависимости кумулянтов Биндера $U_{L}(T)$ для 2D трехвершинной ферромагнитной модели Поттса на решетке Кагоме от температуры для систем с разными линейными размерами $L$ приведены на рис. 5. Как видно из рис. 5 в критической области наблюдается четко выраженная точка пересечения, что и свидетельствует о ФП второго рода. Кроме того, этот рисунок демонстрирует насколько точно можно определить критическую 


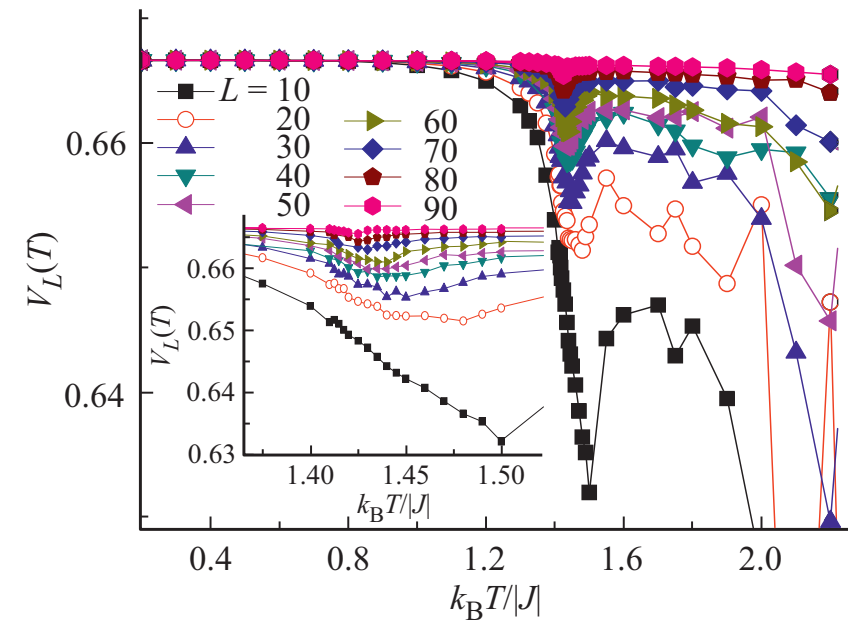

Рис. 6. Температурная зависимость кумулянтов Биндера $V_{L}(T)$ для двумерной трехвершинной модели Поттса на решетке Кагоме. На вставке показано стремление $V_{L}(T)$ к 2/3 с увеличением линейного размера системы $L$ в критической области.

температуру $T_{c}$. На рис. 6 представлены температурные зависимости $V_{L}(T)$. Как видно из вставки на этом рисунке в критической области $V_{L}(T)$ не стремится к нетривиальному значению $V_{L}^{*}(T)$, а стремится к $2 / 3$ с увеличением линейного размера системы $L$, что также характерно для ФП второго рода.

Определенное методом кумулянтов Биндера значение критической температуры $T_{c}$ в единицах $J / k_{\mathrm{B}}$ для двумерной трехвершинной модели Поттса на решетке Кагоме и ее сравнение с аналитическими значениями из литературы приведены в таблице. Как видно из таблицы вычисленное значение $T_{c}$ достаточно хорошо согласуется со значением, полученным с применением низкотемпературного разложения [20] и с применением метода трансфер-матриц [9]. В то же время, это значение заметно отличается от $T_{c}$, полученное в работе $\mathrm{Wu}$ [4], допустив предположение о дуальности решетки Кагоме. При $q=3$ такое предположение дает некорректное значение, отличающееся как от данных метода трансферматриц и низкотемпературного разложения, так и от данных метода Монте-Карло. По-видимому предположение $\mathrm{Wu}$ [4] является верным только для случаев $q=2$ и $q \geq 4$. При $q=2$ модель Поттса на решетке Кагоме имеет точное решение [21].

\section{4. Заключение}

Таким образом, в настоящей работе с соблюдением единой методики, исследована двумерная трехвершинная модель Поттса на решетке Кагоме. На основе метода кумулянтов Биндера четвертого порядка определена критическая температура $T_{c}$ для этой модели и показано, что ее значение достаточно хорошо согласуется со значением критической температуры, полученным как на основе метода трансфер матриц, так и с применением метода низкотемпературного разложения.

\section{Финансирование}

Исследование выполнено при финансовой поддержке РФФИ в рамках научного проекта № 19-02-00153

\section{Конфликт интересов}

Авторы заявляют, что у них нет конфликта интересов.

\section{Список литературы}

[1] F.Y. Wu. Rev. Mod. Phys. 54, 235 (1982).

[2] A.J. Guttmann, I.G. Enting. J. Phys. A 27, 5801 (1994).

[3] M. Loulidi. Physica A 287, 177 (2000).

[4] F.Y. Wu. J. Phys. C 12 (1979).

[5] J.L. Monroe. Phys. Rev. E 67, 017103 (2003).

[6] Q. Chen, S.C. Bae, S. Granick. Nature (London) 469, 381 (2011).

[7] M.Ya. Amusia, K.G. Popov, V.R. Shaginyan, V.A. Stephanovich. Theory of Heavy-Fermion Compounds. Springer International Publishing, Switzerland (2015).

[8] Р. Бэкстер. Точно решаемые модели в статистической механике. Мир, М. (1985). 351 с.

[9] S.K. Baek, H. Makela, P. Minnhagen, B.J. Kim. Phys. Rev. E 83, 061104 (2011).

[10] А.К. Муртазаев, А.Б. Бабаев, Г.Я. Атаева. Физика низких температур 39, 2, 194 (2013).

[11] А.К. Муртазаев, А.Б. Бабаев. Письма в ЖЭТФ 99, 618 (2014) [JETP Lett. 99, 535 (2014)].

[12] А.К. Муртазаев, А.Б. Бабаев. ЖЭТФ 143, 116 (2013) [JETP 116, 101 (2013)].

[13] А.Б. Бабаев, А.К. Муртазаев. Письма в ЖЭТФ 107, 656 (2018) [JETP Letters 107, 624 (2018)].

[14] U. Wolff. Phys. Lett. 62, 361 (1989).

[15] А.К. Муртазаев, И.К. Камилов, А.Б. Бабаев. ЖЭТФ 126, 1377 (2004) [JETP 99, 1201 (2004)].

[16] P. Peczac, A.M. Ferrenberg, D.P. Landau. Phys. Rev. B 43, 6087 (1991).

[17] Y. Saito. J. Phys. A 15, 1885, (1982).

[18] А.Б. Бабаев, А.К. Муртазаев, Э.М. Сулейманов, Т.Р. Ризванова. ФТТ 58, 10, 2001 (2016).

[19] K. Eichhorn, K. Binder. J. Phys.: Condens. Matter 8, 5209 (1996).

[20] H. Feldman, A.J. Guttmann, I. Jensen, R. Shrock, Sh. Tsai. J. Phys. A 31, 2287 (1998).

[21] K. Kanô K., S. Naya. Prog. Theor. Phys. 10, 158 (1953).

Редактор Д.В. Жуманов 